\title{
Cosmological constant decaying with CMB temperature
}

\author{
Tomohide Sonoda \\ Graduate School of Natural Sciences \\ International Christian University \\ 3-10-2 Osawa, Mitaka 181-8585, Tokyo, Japan \\ tomo@alm.icu.ac.jp
}

Received 18 October 2018

Accepted 25 March 2019

Published 18 April 2019

\begin{abstract}
Recent observations of the dark energy density have demonstrated the fine-tuning problem and the challenges faced by theoretical modeling. In this study, we apply the selfsimilar symmetry (SSS) model, describing the hierarchical structure of the universe based on the Dirac large numbers hypothesis, to Einstein's cosmological term. We introduce a new similarity dimension, $D_{B}$, in the SSS model. Using the $D_{B}$ SSS model, the cosmological constant $\Lambda$ is simply expressed as a function of the cosmic microwave background (CMB) temperature. The result shows that both the gravitational constant $G$ and $\Lambda$ are coupled with the CMB temperature, which simplifies the solution of Einstein's field equations for the variable $\Lambda-G$ model.
\end{abstract}

Keywords: Cosmological constant; cosmic microwave background; large numbers hypothesis; self-similarity; symmetry.

Mathematics Subject Classification 2010: 83C20, 83D05, 83F05

\section{Introduction}

The cosmological constant problem, i.e. the dark energy problem, poses a formidable challenge in physics. In 1998, observations of distant supernovae provided evidence of the acceleration of the expansion of the universe [1, 2. Einstein's cosmological term came to be recognized as the simplest candidate to explain the mechanism behind the accelerating universe. However, the inconsistencies between theoretical expectations and observations are extremely problematic, despite many theoretical models being proposed to provide a suitable explanation [3] issue from a different aspect, an axiomatic method has been proposed by Beck [10]. Beck formulated a description of the cosmological constant, $\Lambda$, using four statistical axioms: fundamentality ( $\Lambda$ depends only on the fundamental constants

This is an Open Access article published by World Scientific Publishing Company. It is distributed under the terms of the Creative Commons Attribution 4.0 (CC-BY) License. Further distribution of this work is permitted, provided the original work is properly cited. 
of nature), boundedness ( $\Lambda$ has a lower bound, $0<\Lambda$ ), simplicity ( $\Lambda$ is given by the simplest possible formula, consistent with the other axioms), and invariance $(\Lambda$ values obtained using potentially different values of the fundamental parameters preserve the scale-invariance of the large-scale physics of the universe). Using these four axioms, Beck showed that $\Lambda$ is given as follows:

$$
\Lambda=\frac{G^{2}}{\hbar^{4}}\left(\frac{m_{e}}{\alpha_{f}}\right)^{6},
$$

where $G$ is the gravitational constant, $\hbar$ is the reduced Planck constant, $\alpha_{f}$ is the fine-structure constant, and $m_{\mathrm{e}}$ is the electron mass. The same formula has been proposed using different approaches [11, 12], and has recently been discussed in several reports $13-16$.

While the standard cosmological model assumes that $\Lambda$ is invariant, recent observations in particle physics and cosmology indicate that $\Lambda$ ought to be treated as a dynamical quantity rather than a simple constant 17 28. In addition, dimensional analysis 29] and the action principle [30] suggest that $\Lambda$ and $G$ cannot vary independently. Therefore, a number of cosmological models incorporating variable $\Lambda$ and $G$ have subsequently been studied 31 39.

The result in our earlier study [40], which explains the hierarchical structure of the universe based on the Dirac large numbers hypothesis (LNH) 41, 42, indicates that both $G$ and $m_{e}$ are coupled with the cosmic microwave background (CMB) temperature. In the present study, we applied the previous result to Eq. (11). The new result indicates that $\Lambda$ can be expressed as a function of the CMB temperature. It is well known that $\Lambda$ and $G$ are both important parameters in Einstein's field equations. Our result indicates that $\Lambda$ and $G$ are not independent functions, but are coupled with the CMB temperature. This means that we can simplify the solution of Einstein's field equations for the variable $\Lambda-G$ model, because we can unify the two independent variables $\Lambda$ and $G$ into one simple function of the CMB temperature.

\section{2. $D_{B}$ SSS Model}

The self-similar symmetry (SSS) model 40] describes the CMB with a symmetrical self-similar structure. The model consists of dimensionless values, because a physical constant with a dimension would not have universality [29]. Therefore, we define the fundamental dimensionless mass ratios of the proton mass $m_{\mathrm{pr}}$, electron mass $m_{\mathrm{e}}$, and Planck mass $m_{\mathrm{Pl}}$ as follows:

$$
\begin{aligned}
& A=\log \alpha=\log \left(\frac{m_{\mathrm{Pl}}}{m_{\mathrm{pr}}}\right) \approx 19.11435, \\
& B=\log \beta=\log \left(\frac{m_{e}}{m_{\mathrm{pr}}}\right) \approx-3.26391 .
\end{aligned}
$$

We also define the fundamental dimensionless time and length ratios as follows:

$$
T=\log \left(\frac{t}{t_{\mathrm{Pl}}}\right), \quad L=\log \left(\frac{l}{l_{\mathrm{Pl}}}\right),
$$


where $t$ and $l$ are the time and length scales of objects, respectively, and $t_{\mathrm{Pl}}$ and $l_{\mathrm{Pl}}$ are the Planck time and length, respectively. Using these dimensionless values, we define the similarity dimension $D_{A}$ as

$$
D_{A}=\left(\frac{T}{L}\right)^{3}=\frac{A}{A+B} \approx 1.20592 .
$$

A new similarity dimension, $D_{B}$, is then introduced as follows:

$$
D_{B}=\frac{A-B}{A+B} \approx 1.41184 .
$$

The values of $D_{A}$ and $D_{B}$ correspond to the fractal dimensions for large-scale structures in the universe, reported by several authors $(D=1.2$ 43 46] and $D=1.4$ 47]).

The hierarchical structures of the $D_{B}$ SSS model are constructed according to the following sequences:

$$
\begin{aligned}
& L_{0}=2(A+B) \approx 31.70089, \\
& L_{n}=D_{B}^{n} L_{0} \quad \text { for } L>L_{0}, \\
& L_{m}=\left(2-D_{B}^{m}\right) L_{0} \quad \text { for } L<L_{0},
\end{aligned}
$$

where $n$ and $m$ are natural numbers that represent the hierarchical levels. The time scales of each hierarchy are also calculated using Eq. (44). Equations (7) and (8) indicate that $L_{n}$ and $L_{m}$ are self-similar to $L_{0}$, which corresponds to the CMB temperature [40].

\section{Verification of the $D_{B}$ SSS Model}

To verify the proposed $D_{B}$ SSS model, we compared the model values with reference values. Tables 1 and 2 summarize the length and time scales of the Planck, weak, solar, and universe hierarchies. The values obtained using the $D_{B}$ SSS model closely agree with the reference values. Figure 1 shows the hierarchy time scale as a function of the length scale. The coincidences observed in the figure confirm the validity of the SSS model.

Table 1. Length scales of the hierarchies of the universe.

\begin{tabular}{lcccc}
\hline Hierarchy & $l(\mathrm{~m})$ & $L$ & $D_{B}$ SSS model & Error $(\%)$ \\
\hline Planck scale $^{\mathrm{a}}$ & $1.6 \times 10^{-35}$ & 0 & $0.21(m=2)$ & - \\
Weak scale $^{\mathrm{b}}$ & $10^{-16}$ & 18.79 & $18.65(m=1)$ & -0.8 \\
Solar scale $^{\mathrm{c}}$ & $1.4 \times 10^{9}$ & 43.93 & $44.76(n=1)$ & 1.8 \\
Universe scale $^{\mathrm{d}}$ & $4.1 \times 10^{28}$ & 63.40 & $63.19(n=2)$ & -0.3 \\
\hline
\end{tabular}

a Planck length $l_{\mathrm{P} 1}=\sqrt{\hbar G / c^{3}}$, where $c$ is the speed of light in vacuum.

${ }^{\mathrm{b}}$ Experimental results show that the range of the weak interaction is $r_{w} \leq 10^{-16} \mathrm{~m}$ [48].

${ }^{\mathrm{c}}$ Diameter of the sun, based on the nominal solar radius defined by the International Astronomical Union 49.

${ }^{\mathrm{d}}$ Upper bound of the universe derived from the $D_{A}$ SSS model [40]. 
Table 2. Time scales of the hierarchies of the universe.

\begin{tabular}{lcccc}
\hline Hierarchy & \multicolumn{1}{c}{$t(\mathrm{~s})$} & $T$ & $D_{B}$ SSS model & Error $(\%)$ \\
\hline Planck scale $^{\mathrm{a}}$ & $5.4 \times 10^{-44}$ & 0 & $0.23(m=2)$ & - \\
Weak scale $^{\mathrm{b}}$ & $6.6 \times 10^{-27}$ & 17.09 & $19.85(m=1)$ & 13.9 \\
Solar scale $^{\mathrm{c}}$ & $2.3 \times 10^{5}$ & 48.63 & $47.64(n=1)$ & -2.1 \\
Universe scale $^{\mathrm{d}}$ & $1.7 \times 10^{24}$ & 67.49 & $67.26(n=2)$ & -0.3 \\
\hline
\end{tabular}

a Planck time $t_{\mathrm{Pl}}=\sqrt{\hbar G / c^{5}}$.

${ }^{\mathrm{b}}$ The electromagnetic and weak forces unify at $\left.100 \mathrm{GeV} ; 50\right] t=\hbar / 10^{11} \mathrm{~s}$.

cSun's rotational period; 51] $t=2.32 \times 10^{5} \mathrm{~s}$.

d Time scale of the universe derived from the $D_{A}$ SSS model [40].

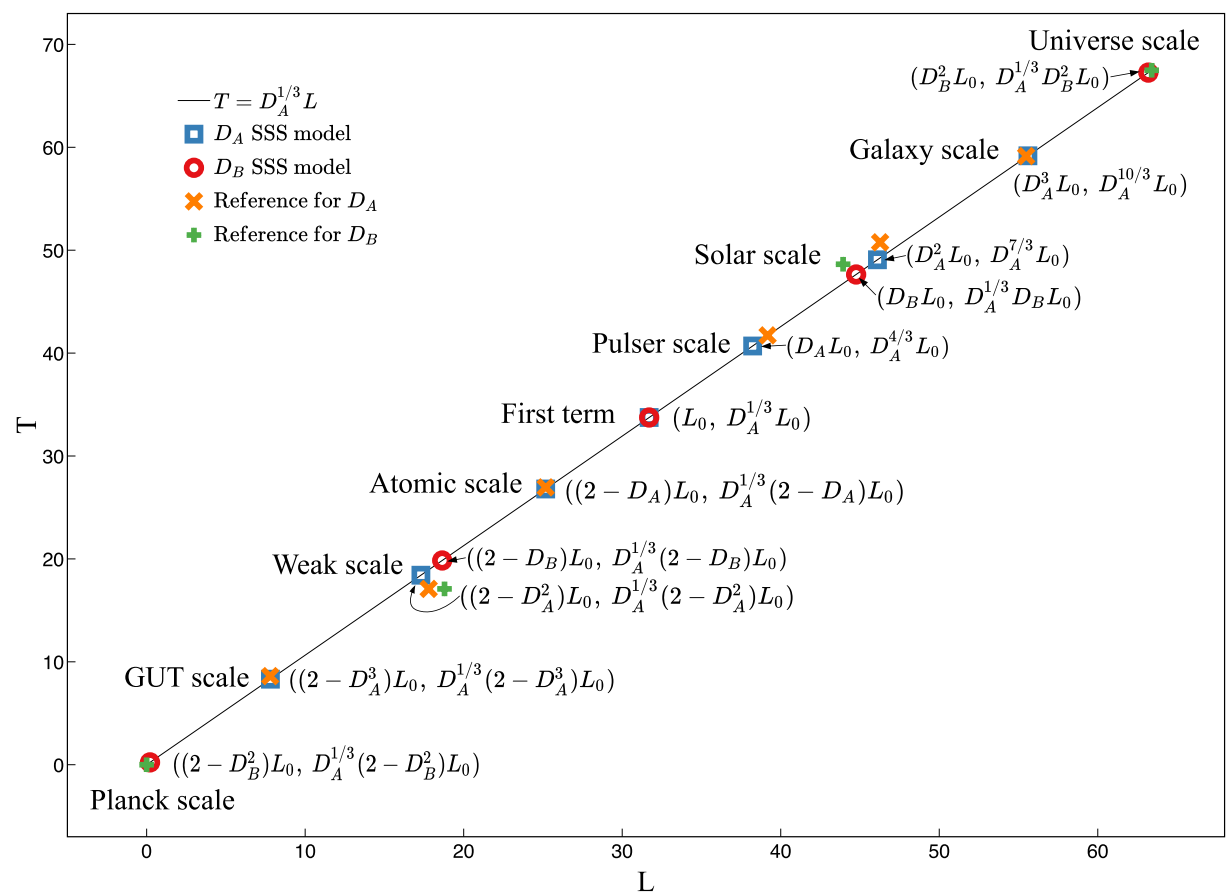

Fig. 1. The time scale as a function of the length scale for the SSS model and reference values. The reference values for the $D_{A}$ SSS model are taken from [40. The lower and upper bounds of the universe are interpolated in the $D_{B}$ SSS model. Note the symmetry of the first term $L_{0}$, which corresponds to the CMB temperature. This symmetry indicates that each hierarchy is self-similar to the CMB temperature.

\section{Discussion}

Using the gravitational coupling constant $\alpha_{G}=G m_{\mathrm{pr}}^{2} / \hbar c$ and Eq. (2), it is obtained that $2 A=-\log \alpha_{G}$. The following relations are satisfied:

$$
\begin{gathered}
L_{n=1}+L_{0}=3 L_{0}-L_{m=1}=4 A \\
L_{m=1}-L_{0}=L_{0}-L_{n=1}=4 B .
\end{gathered}
$$


Therefore, $\alpha_{\mathrm{G}}$ and $\beta$ are important in forming the hierarchical structure of the universe. Regarding the similarity dimension, $D_{A}=\left(r_{a}-r_{b}\right) /\left(1-r_{b}\right)$ (where $r_{a}=$ $\left(D_{A}^{3}+D_{A}^{2}-2\right) / D_{A}$ and $r_{b}=\left(2-D_{A}^{3}\right) /\left(D_{A}^{2}-D_{A}\right)$ are the ratios of the length scales of the hierarchies [40]) can be used to obtain a simple relation between $r_{a}$ and $r_{b}$ :

$$
(\alpha \beta)^{r_{a}}=\alpha \beta^{r_{b}} .
$$

Equation (11) can be interpreted as the basic formula for the similarity dimension, and indicates the correlation between the cosmic structure and fundamental dimensionless mass ratios. ${ }^{a}$ Using Eq. (11), we obtain

$$
D_{B}=\frac{2 r_{a}-r_{b}-1}{1-r_{b}}
$$

However, the numerical relation between $D_{B}$ and $r_{a}$ is

$$
\frac{r_{a} D_{B}}{\sqrt{2}} \approx 1.000009
$$

Equation (13) indicates the validity of $D_{B}$ : if the $D_{B}$ value is substituted into Eq. (8), then $L_{m=2}$ is consistent with the Planck length.

Regarding $\Lambda$, Eq. (1) can be written in an equivalent dimensionless form using $G=\hbar c / m_{\mathrm{Pl}}^{2}$ :

$$
l_{\mathrm{Pl}}^{2} \Lambda=\alpha_{f}^{-6}\left(\frac{m_{e}}{m_{\mathrm{Pl}}}\right)^{6} .
$$

We employed the following formulas derived from the $D_{A}$ SSS model [40] in Eq. (14):

$$
\begin{aligned}
& \alpha_{\mathrm{G}} \simeq \tau_{\mathrm{CMB}}^{D_{A}}, \\
& \beta^{2} \simeq \tau_{\mathrm{CMB}}^{D_{A}-1},
\end{aligned}
$$

where $\tau_{\mathrm{CMB}}=T_{\mathrm{CMB}} / T_{\mathrm{Pl}}$, with $T_{\mathrm{CMB}}$ being the $\mathrm{CMB}$ temperature and $T_{\mathrm{Pl}}$ the Planck temperature. Then, we obtain

$$
\lambda(\xi) \simeq \xi^{3}
$$

where $\lambda$ is the cosmological constant in reduced Planck units, $\lambda=l_{\mathrm{Pl}}^{2} \Lambda$, and we define $\xi \equiv \alpha_{f}^{-2} \alpha_{G}^{D_{B} / D_{A}} \simeq \alpha_{f}^{-2} \tau_{\mathrm{CMB}}^{D_{B}}$. Equation (17) is based on the LNH.

If we employ $T_{\mathrm{CMB}}=2.725 \mathrm{~K}$ as the current parameter in Eq. (17), then we obtain $\lambda_{0} \approx 3.07 \times 10^{-122}$, which is consistent with the latest observational data [52].

If we adopt $T_{\mathrm{CMB}}=T_{\mathrm{Pl}}$ as the initial condition of the universe, and substitute this into Eqs. (15)- (17), then we obtain $\alpha=\beta=1$ and $\lambda_{T_{\mathrm{CMB}}=T_{\mathrm{Pl}}}=\alpha_{f}^{-6}$, which implies that the entire hierarchy was contained in a single point, and that a large $\lambda$ can trigger cosmic inflation. The value of $\lambda$ decreases with the decrease of $T_{\mathrm{CMB}} \ll$ $T_{\mathrm{Pl}}$, while the size of the universe $L$ expands according to $L \propto \log \left(T_{\mathrm{Pl}} / T_{\mathrm{CMB}}\right)$.

a Using Eq. 11, we can derive another similarity dimension $D_{C}=-B /(A+B) \approx 0.20592$. 
Assuming that $T_{\mathrm{CMB}} \rightarrow 0$ is the ultimate fate of the universe, $L \rightarrow \infty$ and $\lambda \rightarrow 0$. This indicates that the universe falls into an inactive state as it expands to infinity.

\section{Conclusions}

We showed that the similarity dimension $D_{B}$ based on the SSS model is valid for describing the hierarchy structure of the universe. Using $D_{B}, \Lambda$ is expressed as a function of the CMB temperature. Linde [17 proposed that $\Lambda$ is a function of temperature, and related it to the process of broken symmetries. Gasperini [20, 21] also argued that $\Lambda$ can be interpreted as a measure of the temperature of the vacuum. Our result supports their arguments in this respect. The cosmological scenario of a dynamically decaying $\Lambda$ gives a natural interpretation for sufficiently small $\Lambda$ in the present epoch. Taking Eq. (17) together with Eq. (15), we can unify the two parameters $\Lambda$ and $G$ in Einstein's field equations as a single parameter of the CMB temperature. We should apply this result to Einstein's field equations to analyze further details in the future.

\section{Acknowledgments}

The author thanks M. B. Greenfield and K. Kitahara for the helpful discussions.

\section{References}

[1] A. G. Riess, A. V. Filippenko, P. Challis, A. Clocchiatti, A. Diercks, P. M. Garnavich, R. L. Gilliland, C. J. Hogan, S. Jha, R. P. Kirshner et al., Observational evidence from supernovae for an accelerating universe and a cosmological constant, Astron. J. 116 (1998) 1009.

[2] S. Perlmutter, G. Aldering, G. Goldhaber, R. Knop, P. Nugent, P. Castro, S. Deustua, S. Fabbro, A. Goobar, D. Groom et al., Measurements of $\Omega$ and $\Lambda$ from 42 highredshift supernovae, Astrophys. J. 517 (1999) 565.

[3] S. Weinberg, The cosmological constant problem, Rev. Mod. Phys. 61 (1989) 1.

[4] V. Sahni and A. Starobinsky, The case for a positive cosmological $\Lambda$-term, Int. J. Mod. Phys. D 9 (2000) 373.

[5] S. M. Carroll, The cosmological constant, Living Rev. Relativ. 4 (2001) 1.

[6] T. Padmanabhan, Cosmological constant the weight of the vacuum, Phys. Rep. $\mathbf{3 8 0}$ (2003) 235.

[7] P. J. E. Peebles and B. Ratra, The cosmological constant and dark energy, Rev. Mod. Phys. 75 (2003) 559.

[8] E. J. Copeland, M. Sami and S. Tsujikawa, Dynamics of dark energy, Int. J. Mod. Phys. D 15 (2006) 1753.

[9] K. Bamba, S. Capozziello, S. Nojiri and S. D. Odintsov, Dark energy cosmology: the equivalent description via different theoretical models and cosmography tests, Astrophys. Space Sci. 342 (2012) 155.

[10] C. Beck, Axiomatic approach to the cosmological constant, Phys. A: Stat. Mech. Appl. 388 (2009) 3384.

[11] L. Nottale, Mach's Principle, Dirac's Large Numbers, and the Cosmological Constant Problem, preprint (1993). 
[12] C. Boehmer and T. Harko, Physics of dark energy particles, Found. Phys. 38 (2008) 216.

[13] P. Burikham, K. Cheamsawat, T. Harko and M. J. Lake, The minimum mass of a charged spherically symmetric object in D dimensions, its implications for fundamental particles, and holography, Eur. Phys. J. C 76 (2016) 106.

[14] L. Eaves, The apparent fine-tuning of the cosmological, gravitational and fine structure constants, Phys. A: Stat. Mech. Appl. 443 (2016) 355.

[15] H. Wei, X.-B. Zou, H.-Y. Li and D.-Z. Xue, Cosmological constant, fine structure constant and beyond, Eur. Phys. J. C 77 (2017) 14.

[16] M. J. Lake, Is there a connection between "dark" and "light" physics? J. Phys. Con. Ser. 883 (2017) 012001.

[17] A. D. Linde, Is the cosmological constant a constant? JETP Lett. 19 (1974) 183.

[18] M. Özer and M. Taha, A possible solution to the main cosmological problems, Phys. Lett. B 171 (1986) 363.

[19] K. Freese, F. C. Adams, J. A. Frieman and E. Mottola, Cosmology with decaying vacuum energy, Nucl. Phys. B 287 (1987) 797.

[20] M. Gasperini, Decreasing vacuum temperature: A thermal approach to the cosmological constant problem, Phys. Lett. B 194 (1987) 347.

[21] M. Gasperini, A thermal interpretation of the cosmological constant, Class. Quantum Gravit. 5 (1988) 521.

[22] A.-M. Abdel-Rahman, A critical density cosmological model with varying gravitational and cosmological constants, Gen. Relativ. Gravit. 22 (1990) 655.

[23] Y. Fujii and T. Nishioka, Model of a decaying cosmological constant, Phys. Rev. D 42 (1990) 361.

[24] W. Chen and Y.-S. Wu, Implications of a cosmological constant varying as $R^{-2}$, Phys. Rev. D 41 (1990) 695.

[25] M. S. Berman, Cosmological models with a variable cosmological term, Phys. Rev. D 43 (1991) 1075.

[26] J. Carvalho, J. Lima and I. Waga, Cosmological consequences of a time-dependent $\Lambda$ term, Phys. Rev. D 46 (1992) 2404.

[27] D. Kalligas, P. Wesson and C. Everitt, Flat FRW models with variable $G$ and $\Lambda$, Gen. Relativ. Gravit. 24 (1992) 351.

[28] J. Overduin and F. Cooperstock, Evolution of the scale factor with a variable cosmological term, Phys. Rev. D 58 (1998) 043506.

[29] A. Narimani, A. Moss and D. Scott, Dimensionless cosmology, Astrophys. Space Sci. 341 (2012) 617.

[30] K. Krori, S. Chaudhury and A. Mukherjee, Cosmologies with variable $G$ and $\Lambda$ from action principle, Gen. Relativ. Gravit. 32 (2000) 1439.

[31] A. I. Arbab, Cosmological consequences of a built-in cosmological constant model, J. Cosmol. Astropart. Phys. 2003 (2003) 008.

[32] R. F. Sisteró, Cosmology with $G$ and $\Lambda$ coupling scalars, Gen. Relativ. Gravit. 23 (1991) 1265.

[33] R. Vishwakarma et al., Some FRW models with variable $G$ and $\Lambda$, Class. Quantum Gravit. 14 (1997) 945.

[34] T. Harko and M. Mak, Particle creation in cosmological models with varying gravitational and cosmological constants, Gen. Relativ. Gravit. 31 (1999) 849.

[35] A. Pradhan and V. K. Yadav, Bulk viscous anisotropic cosmological models with variable $G$ and $\Lambda$, Int. J. Mod. Phys. D 11 (2002) 893.

[36] J. Singh, A. Pradhan and A. K. Singh, Bianchi type-I cosmological models with variable $G$ and $\Lambda$-term in general relativity, Astrophys. Space Sci. 314 (2008) 83. 
[37] C. Singh and A. Beesham, Particle creation in higher dimensional space-time with variable $G$ and $\Lambda$, Int. J. Theor. Phys. 51 (2012) 3951.

[38] L. Kantha, A time-dependent and cosmological model consistent with cosmological constraints, Adv. Astron. 2016 (2016) 9743970, 8 pages.

[39] R. Tiwari, A. Beesham, R. Singh and L. Tiwari, Time varying $G$ and $\Lambda$ cosmology in $f(R, T)$ gravity theory, Astrophys. Space Sci. 362 (2017) 143.

[40] T. Sonoda, Self-similar symmetry model and cosmic microwave background, Front. Appl. Math. Stat. 2 (2016) 5.

[41] P. A. Dirac, The cosmological constants, Nature 139 (1937) 323.

[42] P. A. Dirac, A new basis for cosmology, in Proc. of the Royal Society of London A: Mathematical, Physical and Engineering Sciences, Vol. 165 (Royal Society, 1938), pp. 199-208.

[43] A. S. Szalay and D. N. Schramm, Are galaxies more strongly correlated than clusters? Nature 314 (1985) 718.

[44] L. Pietronero, The fractal structure of the Universe: Correlations of galaxies and clusters and the average mass density, Phys. A: Stat. Mech. Appl. 144 (1987) 257.

[45] N. A. Bahcall, Large-scale structure in the universe indicated by galaxy clusters, Ann. Rev. Astron. Astrophys. 26 (1988) 631.

[46] L. Guzzo, A. Iovino, G. Chincarini, R. Giovanelli and M. P. Haynes, Scale-invariant clustering in the large-scale distribution of galaxies, Astrophys. J. 382 (1991) L5.

[47] E. M. de Gouveia dal Pino, A. Hetem, J. E. Horvath, C. A. de Souza, T. Villela and J. de Araujo, Evidence for a very large scale fractal structure in the universe from COBE measurements, Astrophys. J. 442 (1995) L45.

[48] J. Barrow and F. J. Tipler, The Anthropic Cosmological Principle (Oxford University, New York, 1986), pp. 294.

[49] A. Prša, P. Harmanec, G. Torres, E. Mamajek, M. Asplund, N. Capitaine, J. Christensen-Dalsgaard, É. Depagne, M. Haberreiter, S. Hekker et al., Nominal values for selected solar and planetary quantities: IAU 2015 Resolution B3, Astron. J. 152 (2016) 41.

[50] G. Bertone, D. Hooper and J. Silk, Particle dark matter: Evidence, candidates and constraints, Phys. Rep. 405 (2005) 279.

[51] National Astronomical Observatory of Japan, Chronological Scientific Tables (Maruzen Co., Ltd, 2016), pp. 97.

[52] P. A. Ade, N. Aghanim, M. Arnaud, M. Ashdown, J. Aumont, C. Baccigalupi, A. Banday, R. Barreiro, J. Bartlett, N. Bartolo et al., Planck 2015 results-XIII. Cosmological parameters, Astron. Astrophys. 594 (2016) A13. 